\title{
Diffusion of Particles, Heat and Magnetic Fields in Compressible Turbulent Media
}

\author{
A.Z. Dolginov and N.A. Silant'ev \\ A.I.Ioffe Phys.-Tech. Institute \\ Academy of Sciences of USSR \\ 194021 Leningrad, USSR
}

\begin{abstract}
A new method for the calculation of kinetic coefficients is presented. This method allows us to obtain the distribution of scalar and vector fields (such as the temperature, the admixture particle number density and the magnetic field) in turbulent cosmic media with any value of $S=u_{0} \tau_{0} / R_{0}$. The explicit expression for the "turbulent" diffusivity $D_{T}$ is obtained. In some cases $D_{T}$ becomes negative, implying the clustering of the admixture particles in patches (a local increase of the temperature and magnetic fields). The magnetic $\alpha$-effect is considered for the case $S \sim 1$.
\end{abstract}

Processes of scalar and vector field transport in turbulent medium are of great importance in most astrophysical objects. The general form of the equations which describe these processes is

$$
\hat{K} f(\boldsymbol{r}, t)=\hat{L} f(\boldsymbol{r}, t), \quad \hat{K}=\frac{\partial}{\partial t}-D_{0} \nabla^{2} .
$$

(a) For the case of particle propagation $f(\boldsymbol{r}, t)=f_{p}(\boldsymbol{r}, t)$ is the number density of the particles with momentum $p$ at the space-time point $r, t$, and $\hat{L}=\hat{L}_{p}=-(\boldsymbol{u}) \nabla-\operatorname{div} \boldsymbol{u}(p / 3)(\partial / \partial p) \cdot \boldsymbol{u}(\boldsymbol{r}, t)$ is the turbulent velocity of the medium. $\langle u(r, t)\rangle=0$. The brackets \langle\rangle denote an averaging over the velocity ensemble.

(b) If one considers the particle distribution, disregarding their momenta, then $f(\boldsymbol{r}, t)=N(\boldsymbol{r}, t)=\int_{0}^{\infty} f_{p}(\boldsymbol{r}, t) p^{2} d p$ and $\hat{L}=\hat{L}_{N}=-(\boldsymbol{u} \nabla)-\operatorname{div} \boldsymbol{u}$.

(c) For the case of heat propagation $f(\boldsymbol{r}, t)=T(\boldsymbol{r}, t), \hat{L}=\hat{L}_{T}=-(\boldsymbol{u} \nabla)-$ $\mathcal{R} / \mu C_{V} \operatorname{div} u$,where $T$ is the temperature, $\mu$ is the molecular weight, $C_{V}$ is the specific heat capacity and $\mathcal{R}$ is the gas constant.

(d) The magnetic field distribution is determined by (1) with $f(\boldsymbol{r}, t)=\boldsymbol{B}(\boldsymbol{r}, t)$, the magnetic field, and $\hat{L} f=\hat{\mathcal{L}}_{B} \boldsymbol{B}=-(\boldsymbol{u} \nabla) \boldsymbol{B}+(\boldsymbol{B} \nabla) \boldsymbol{u}-\boldsymbol{B} \operatorname{div} \boldsymbol{u} . D_{0}$ in $(1)$ is the diffusion coefficient for the field considered.

Most papers devoted to the solution of (1) are restricted to the case $S^{2}=$ $\left(u_{0} \tau_{0} / R_{0}\right)^{2} \ll 1$, where $u_{0}^{2}=\left\langle u^{2}(\boldsymbol{r}, t)\right\rangle / 3$ and $R_{0}$ and $\tau_{0}$ are the space and time 
scales of the turbulence. By definition the motion within the scale values of order $R_{0}, \tau_{0}$ is more or less regular and becomes random only for scale sizes $L \gg R_{0}$ and $t \gg \tau_{0}$. The condition $S^{2} \ll 1$ allows us to take into account only the correlation tensor of second rank, $\left\langle u_{i}(\boldsymbol{r}, t) u_{j}(\boldsymbol{r}, t)\right\rangle$ where $\boldsymbol{R}=\boldsymbol{r}-\boldsymbol{r}^{\prime}, \tau=t-t^{\prime}$. The turbulence is assumed to be stationary and homogeneous. If $S Z 1$ the correlation tensors of higher rank become important.

In this paper we present a new method for the solution of the transport problem in a compressible turbulent media for the case $S \gtrless 1$. The Greens function of (1) is determined by

$$
(\hat{K}-\hat{L}) G(1,2)=\delta(1-2) \equiv \delta\left(\boldsymbol{r}_{1}-\boldsymbol{r}_{2}\right) \delta\left(t_{1}-t_{2}\right),
$$

where the symbols $1,2, \ldots$ denote $\boldsymbol{r}_{1}, t_{1} ; \boldsymbol{r}_{2}, t_{2} ; \ldots$ and $\mathrm{d} 1, \mathrm{~d} 2, \ldots$ denote $d \boldsymbol{r}_{1} d t_{1}$ $; d \boldsymbol{r}_{2} d t_{2} ; \ldots$ The integral form of $(2)$ is

$$
G(1,2)=G_{0}(1,2)+\int \mathrm{d} 3 G_{0}(1,3) \hat{L}(3) G(3,2), \quad \hat{K} G_{0}(1,2)=\delta(1,2) .
$$

If one uses a direct iteration of (3), beginning with $G_{0}(1,2)$, then a divergent series for the case $S \gtrsim 1$ is obtained. The series is valid and asymptotic for $S^{2} \ll 1$. To avoid this difficulty let us take instead of (3) the two coupled equations (4) and (5), which are identical to equation (3) for any value of the operator $\hat{O}$. The particular choice of $\hat{O}$ is equivalent to the particular choice of the zero approximation for $G(1,2)$.

$$
\begin{gathered}
G(1,2)=M(1,2)+\int \mathrm{d} 3 G(1,3)\left[\hat{L}(3) M(3,2)-\int \mathrm{d} 4 \hat{O}(3,4) M(4,2)\right], \\
M(1,2)=G_{0}+\int \mathrm{d} 3 \int \mathrm{d} 4 G_{0}(1,3) \hat{O}(3,4) M(4,2) .
\end{gathered}
$$

The advantage of this form is that it allows the possibility of choosing the zero approximation in such a way to provide a better convergence of the iteration and it takes into account the higher rank correlation tensors. Let us choose $\hat{O}(3,4)=\left\langle\hat{L}(3) G_{0}(3,4) \hat{L}(4)\right\rangle$, leading to an asymptotic iteration series for $G(1,2)$ adequate for $S^{2} \sim 1$. This statement can be proved by calculations with subsequent approximations. The second iteration takes into account the correlation tensors up to the fourth rank, etc. The equation for the mean Greens function is

$$
\langle G(1,2)\rangle=G_{0}(1,2)+\int \mathrm{d} 3 G_{0}(1,3)\langle\hat{L}(3) G(3,2)\rangle .
$$

Equations (5) and (6) are approximately equivalent if one inserts (3) into (6) and takes $\left\langle\hat{L}(3) G_{0}(3,4) \hat{L}(4)\right\rangle\left\langle G(4,2)\right.$ instead of $\left\langle\hat{L}(3) G_{0}(3,4) \hat{L}(4) G(4,2)\right\rangle$. This approximation for $M(1,2)$ is, in many cases, sufficiently precise $(\sim 10 \%)$ for large values of $S^{2} . G(1,2)$ depends on the particular form of $\hat{L}$. Consider the case $\hat{L}=\hat{L}_{N}=-(\boldsymbol{u} \nabla)-\operatorname{div} \boldsymbol{u}$, as an example. Taking into account that $\langle G(R, \tau)\rangle$ is a smooth function on scales $\sim R_{0}, \tau_{0}$, one can take $\langle G\rangle$ out of the integral. This leads to a diffusion equation for $N(\boldsymbol{r}, t)$ with a "turbulent" diffusivity: 


$$
\begin{aligned}
& \partial N / \partial t-\left(D_{0}+D_{T}\right) \nabla^{2} N=0 \\
& D_{T}=\frac{1}{3} \int d \boldsymbol{R} \int d \tau\left[\left\langle u_{i}(1) G(1,2) u_{i}(2)\right\rangle-\boldsymbol{R} \cdot\langle\boldsymbol{u}(1) G(1,2) \operatorname{div} \boldsymbol{u}(2)\rangle\right]
\end{aligned}
$$

In the first approximation we can take in $(7) G(1,2) \approx M(R, \tau)$. The kernel of (5) depends on $\boldsymbol{R}$ and $\tau$ only and $M(R, \tau)$ can be obtained in the explicit form using the Fourier transform. Consider, as a simple example, the potential turbulence $\boldsymbol{u}=\nabla \varphi$ with the correlation function

$$
\langle\varphi(1) \varphi(2)\rangle=\frac{3 u_{0}^{2}}{k_{0}^{2}} \frac{\sin k_{0} R}{k_{0} R} \exp \left(-\frac{|\tau|}{\tau_{0}}\right) .
$$

In this case the Fourier transform of the $M(R, \tau)$ is

$$
\tilde{M}(\boldsymbol{k}, \tau)=\frac{\theta(\tau)}{a}\left[\exp \left(-\frac{\tau}{2 \tau_{0}}\right)\right]\left[\sin \frac{a \tau}{2 \tau_{0}}+a \cos \frac{a \tau}{2 \tau_{0}}\right],
$$

where $\theta(\tau)=1$ if $\tau>0$ and $\theta(\tau)=0$ if $\tau<0, a=\left(4 u_{0}^{2} \tau_{0}^{2}-1\right)^{1 / 2}$ and $D_{0}$ is assumed to be much smaller than $D_{T}$. The first integral term in (7) is always positive, but the sign of the second one depends on the particular form of $\langle u G d i v u\rangle$. In a compressible medium ( $\operatorname{div} \boldsymbol{u} \neq 0$ ) $\operatorname{div} \boldsymbol{u}$ may be negative. Using (8), (9) and (7) we obtain

$$
D_{T}=2 u_{0}^{2} \tau_{0}\left(2-9 S^{2}\right)\left(2+9 S^{2}\right)^{-2}, \quad S=u_{0} \tau_{0} k_{0} / 3,
$$

and so $D_{T}$ becomes negative if $S^{2}>2 / 9$. There are cases, for example acoustic turbulence, when $D_{T}+D_{0}$ is always positive. The negative diffusion leads to formation of patches of the admixture particles. It does not contradict the laws of thermodynamics, because the physical system is thermodynamically open.

By the method described above we can obtain the equation for the average magnetic field in the case $S \gtrsim 1$. We obtain

$$
\left(\frac{\partial}{\partial t}-\left(D_{0}+D_{T}\right) \nabla^{2}\right)\langle B\rangle=\alpha_{T} \operatorname{rot}\langle B\rangle-\beta_{T} \frac{\partial}{\partial t} \operatorname{rot}\langle B\rangle .
$$

In the case of isotropic, stationary, homogeneous non-mirror symmetric turbulence

$$
\begin{gathered}
\left\langle u_{i}(1) u_{j}(2)\right\rangle=\delta_{i j} B_{\perp}(R, \tau)+R_{i} R_{j}\left(B_{\|}(R, \tau)-\right. \\
\left.B_{\perp}(R, \tau)\right) R^{-2}+e_{i j k} R_{k} C(R, \tau) .
\end{gathered}
$$

In this case $D_{T}$ and $\alpha_{\tau}$ are given by

$$
\begin{aligned}
D_{T}= & -\frac{1}{6 \pi^{2}} \int_{0}^{\infty} d q q^{3} \int_{0}^{\infty} d \tau\left[\frac{1}{2}\left(\tilde{M}_{+}+\tilde{M}_{-}\right) \frac{\partial}{\partial q} \tilde{B}_{\|}(q, \tau)-\right. \\
& \left.\left(\tilde{M}_{+}-\tilde{M}_{-}\right) \frac{1}{q} \frac{\partial}{\partial q} \hat{C}(q, \tau)\right], \\
\alpha_{T}= & \frac{1}{3(2 \pi)^{3}} \int d q \int_{0}^{\infty} d \tau\left[\frac{1}{2 q}\left(\tilde{M}_{+}+\tilde{M}_{-}\right)\left(\delta_{i j} q^{2}+q_{i} q_{j}\right) \tilde{B}_{i j}(\boldsymbol{q}, \tau)-\right.
\end{aligned}
$$




$$
\begin{aligned}
& \left.q\left(\tilde{M}_{+}-\tilde{M}_{-}\right) \frac{\partial}{\partial q} \tilde{C}(q, \tau)\right] \\
& B_{i j}(\boldsymbol{R}, \tau)=\left\langle u_{i}(1) u j(2)\right\rangle .
\end{aligned}
$$

The expression for $\alpha_{T}$ differs from that for $\beta_{T}$ only by an additional factor $\tau^{-1}$ in the integral.

$$
\begin{aligned}
\tilde{M}_{ \pm}(q, \tau) & =\left[a_{ \pm}^{-1} \sin \left(a_{ \pm} \tau / 2 \tau_{0}\right)+\cos \left(a_{ \pm} \tau / 2 \tau_{0}\right)\right] \exp \left(-\tau / 2 \tau_{0}\right) \theta(\tau) \\
a_{ \pm} & =\left(4 u_{0}^{2} \tau_{0}^{2} q^{2}-1 \pm 8 C_{0} \tau_{0}^{2} q\right)^{1 / 2} \\
C(0, \tau) & =C_{0} \exp \left(-|\tau| / \tau_{0}\right) .
\end{aligned}
$$

In an incompressible medium with $B_{\|}(0, \tau)=u_{0}^{2} \exp \left(-|\tau| / \tau_{0}\right), c_{0}=\gamma u_{0}^{2} k_{0} / 2$, $|\gamma| \leq 1, S=u_{0} \tau_{0} k_{0} / 3$ we have

$$
D_{T}=\frac{2 u_{0}^{2} \tau_{0}\left(2+9\left(1+\gamma^{2}\right) S^{2}\right)}{\left(2+9 S^{2}\right)^{2}-81 \gamma^{2} S^{4}}, \quad \alpha_{T}=\frac{4 u_{0}^{2} k_{0}\left(1+9 S^{2}\right) \gamma}{\left(2+9 S^{2}\right)-81 \gamma^{2} S^{4}}
$$

Equations (15) are valid to within an error of $\sim 20 \%$ up to $S=1$.

\section{References}

Dolginov, A.Z., Silant'ev, N.A.: 1987, J. Exp. Theor. Phys. JETP 93, 159

Dolginov, A.Z., Silant'ev, N.A.: 1990, Astron. Astrophys.

Dolginov, A.Z., Sliant'ev, N.A.: 1991, Geoph. Astroph. Fluid Dynamics (submitted)

Silant'ev, N.A., Korolkov, M.D.: 1990, Astron. Nachr. 311, 107

Silant'ev, N.A.: 1988, Proc. Int. Meet. on "Physics and Evolution of Stars", Leningrad, Nauka 